\title{
EXCLUSIÓN SOCIAL Y EDUCACIÓN SUPERIOR: LA RESPUESTA PEDAGÓGICA
}

\author{
DÍAZ-TORRES, Juan Manuel ${ }^{1^{*}}$ \\ ${ }^{1}$ Universidad de La Laguna \\ jmdiazt@ull.es*
}

\section{RESUMEN}

Los desafíos que afrontan los educadores sociales en el mundo actual requieren una revisión exhaustiva del papel estratégico de la educación superior. En esta tarea, será necesario explorar nuevos tipos de estrategias capaces de proporcionar una respuesta eficaz a las nuevas necesidades de trabajo social, tales como el diseño del cambio, la innovación, la diversidad cultural y la complejidad social. La promoción del trabajo en equipo, la mejora de habilidades lingüísticas y la aplicación de la resolución de problemas son fundamentales. Estas competencias se ejecutan en las discusiones realizadas por equipos de debate. La exclusión social exige una respuesta pedagógica. En efecto, este trabajo tiene como objetivos analizar el potencial de los equipos de trabajo y sus ventajas para la adaptación a los entornos sociales inciertos así como examinar los efectos que la dinámica de los procesos colaborativos puede tener sobre los procesos de grupo y los resultados académicos.

PALABRAS CLAVE: Práctica pedagógica. Pedagogía social. Educación superior. Exclusión social.

\section{EXCLUSÃO SOCIAL E ENSINO SUPERIOR: A RESPOSTA PEDAGÓGICA}

\section{RESUMO}

Os desafios enfrentados pelos educadores sociais no mundo de hoje exigem uma revisão exaustiva do papel estratégico do ensino superior. Nessa tarefa, será necessário explorar novos tipos de estratégias capazes de dar resposta efetiva às novas necessidades de trabalho social, como o design da mudança, a inovação, a diversidade cultural e a complexidade social. A promoção do trabalho em equipe, a melhoria das competências linguísticas e a implementação da resolução de problemas são importantes. Essas habilidades e competências são executadas em discussões em equipe. A exclusão social exige uma resposta educativa. Com efeito, este trabalho visa analisar o potencial de equipes de trabalho e suas vantagens para se adaptarem a ambientes sociais incertos, bem como examinar os efeitos que a dinâmica das atividades e os processos colaborativos podem ter nos processos de grupo e nos resultados acadêmicos.

PALAVRAS-CHAVE: Prática pedagógica. Pedagogia social. Ensino superior. Exclusão social.

\section{SOCIAL EXCLUSION AND HIGHER EDUCATION: THE PEDAGOGICAL RESPONSE}

\begin{abstract}
The challenges facing social educators in today's world require an exhaustive review of the strategic role of higher education. In this task, it will be necessary to explore new types of strategies capable of providing an effective response to new social work needs, such as the design of change, innovation, cultural diversity and social complexity. The promotion of team work, the improvement of language skills and the implementation of

the resolution of problems are important. These skills and competencies are executed in team discussions. Social exclusion requires an educational response. In effect, this paper aims, first, to analyze the potential of work teams and their advantages for adapting to uncertain social environments. And secondly, to examine the effects that activities dynamics and the collaborative processes may have on group processes and academic outcomes.
\end{abstract} KEYWORDS: Teaching practice. Social work. Higher education. Social exclusion. 


\section{LA EXCLUSIÓN COMO ALEJAMIENTO DE LA DINÁMICA SOCIAL}

Hablar de exclusión social es poner de manifiesto en qué medida se deja de tener un lugar en la sociedad. En efecto, la exclusión social es un proceso paulatino, largo, complejo, pluridimensional, multicausal y acumulativo, que afecta, inferiorizando y alejando, tanto a personas tomadas individualmente como a grupos sociales (DE OÑA, 2009; ESTIVILL, 2003). Dicho proceso marca la distancia entre los que son excluidos e ignorados y aquellos otros que participan de su dinámica y se benefician de ella.

Además de ello, el hecho de la exclusión social como situación que sufren personas o grupos sociales que son condenados a la indigencia generalizada y al aislamiento social también es un fenómeno multidimensional, atendiendo a su génesis. En efecto, no puede obviarse el hecho de que la exclusión social es el resultado de un proceso en el que están imbricados factores de carácter económico, cultural y sociopolítico.

Atendiendo a su expresión social, puede hallarse tras este proceso de ruptura social y de expulsión de los espacios sociales normalizados una multiplicidad de rostros humanos, cuya posibilidad de reconstruir una identidad positiva queda muy alejada de poder ser lograda por su propio esfuerzo. Entre estos, se halla no solo el rostro del minusválido, del niño abandonado, del joven desescolarizado o que fracasa en sus estudios, del toxicómano, del discapacitado sin recursos y del alcohólico; también, de quien acusa una notable precariedad propia e interior inherente, de quien carece de asistencia sanitaria, del enfermo, del inmigrante, del indigente, del parado de larga duración con cierta edad y que arrastra una enorme dificultad de integración laboral. En ese colectivo también podría hallarse el rostro del recluso y de quien ha dejado de serlo, de quien carece de ingresos económicos suficientes, de quien tiene problemas de acceso a unos mínimos educativos y de quien pertenece a una minoría étnica.

¿Y qué decir de quien tiene dificultades insalvables para acceder a la justicia, de la mujer y del niño que malviven en situación de violencia familiar o de pobreza, de la víctima de la trata de personas, de la persona mayor sin recursos expuesta, por tanto, al aislamiento o a la dependencia, de quien carece de una vivienda digna, de quien no tiene apoyos familiares y, por último, del refugiado? Tales rostros muestran la oscuridad de la marginación definitiva, el orco

Educação \& Formação, Fortaleza, v. 2, n. 6, p. 3-16, set./dez. 2017

DOI: http://dx.doi.org/10.25053/edufor.v2i6.2379

http://seer.uece.br/redufor 
del aislamiento comunitario, así como la lenta expiración social del sujeto inmerso en ella (CARMONA, 2005; LÓPEZ, 2005).

\section{EL TRABAJO PEDAGÓGICO CON PERSONAS EN SITUACIÓN DE EXCLUSIÓN}

El trabajo en situaciones complejas, complicadas y con personas en situación vulnerable requiere unas capacidades específicas, tales como la empatía, las aptitudes para entablar relaciones personales sanas y la aptitud para fomentar actitudes sociales. Además de ellas, requiere también gran madurez, elevado equilibrio personal, destreza intelectual proclive al análisis de realidades tanto personales como sociales, una profunda implicación en su labor y una enorme capacidad para trabajar en equipos interdisciplinares.

Pues bien, la presente actividad formativa se ha llevado a cabo en el marco de la docencia universitaria del Grado en Pedagogía y ha estado dirigida a la formación en competencias y capacidades de quienes se forman en la educación superior para dedicar sus esfuerzos a la mejora social de los colectivos más vulnerables y desfavorecidos, así como al desarrollo humano en todas sus facetas.

Inicialmente se propuso probar que la práctica institucionalizada del debate por equipos en el aula es una herramienta útil y necesaria para la cualificación del futuro pedagogo preocupado por el proceso de la exclusión social, así como un instrumento eficaz en la configuración de su perfil psicológico. A su vez, se trató de evidenciar que los efectos formativos obtenidos tras su ejecución permitieran afirmar la consecución de una apreciable mejoría en las dimensiones cognitiva y ética del estudiantado universitario participante. Se trató, en definitiva, de un tipo de acción formativa, tanto teórica como práctica, vinculada a la atención a los colectivos en situación de grave dificultad social.

\section{EL MARCO TEÓRICO DE LA INTERVENCIÓN SOCIOEDUCATIVA}

Investigaciones recientes sobre el fenómeno de la exclusión social han permitido ir perfilando sus características esenciales (APOSPORI; MILLAR, 2003; FERNÁNDEZ, 2013; FISCHER, 2011; SILVER, 2007; TEZANOS et al., 2013; TSAKLOGLOU; PAPADOPOULOS, 2002). 
El itinerario lento, progresivo, largo, complejo, multicausal y acumulativo (DE HAAN, 1999; SUBIRATS, 2005) que es la exclusión social termina menoscabando la dignidad tanto de personas como de grupos sociales, alejándolos de su entorno social (DE OÑA, 2009; ESTIVILL, 2003). De ahí que la intervención socioeducativa del educador social especializado se dirija, primordialmente, a enseñar y capacitar a las personas más vulnerables, marginadas o inmersas en un proceso de exclusión social. De lo que se trata es de dotar a cada uno de los destinatarios de aquellos conocimientos, habilidades y competencias que favorezcan su retorno a la zona social normalizada y su integración plena y definitiva en ella.

Debe indicarse que el educador social especializado es un profesional de la educación, de atención directa, que trabaja en el marco de la vida cotidiana con sujetos, grupos y comunidades, los cuales, por diversas causas que pueden ser de orden físico, psíquico o social, se encuentran en situaciones de riesgo, de dificultad consigo mismo o con el entorno inmediato. Dicha definición incluyó el hecho de que dicho educador tiene como objetivo la potenciación del desarrollo de aquellos recursos del sujeto que faciliten su inserción social activa y su inclusión en un entorno comunitario ampliado.

El educador social especializado es aquel que con su intervención hace protagonistas a sus destinatarios de una acción social que pretende modificar determinadas situaciones personales y sociales, de riesgo social o de marginación, a través de estrategias eminentemente educativas. Así, el educador especializado pretende que los destinatarios de su labor tomen conciencia de su propia identidad y, por supuesto, de su dignidad personal, a fin de proporcionar a cada uno de ellos la posibilidad de mejorar su propia calidad de vida (LÓPEZ, 2005).

Dicho educador tiene como cometido proporcionar recursos suficientes para la autoconcienciación y para la realización de una nueva e ilusionante planificación vital, todo ello en un marco en el que los destinatarios se sientan - y sean - protagonistas de sus propios procesos existenciales y responsables de sus propios destinos vitales. Tales recursos son, en el fondo, habilidades o destrezas sociales e instrumentales destinadas a incrementar la capacidad individual en general y el potencial para la interacción comunitaria.

Saber orientar, saber cómo facilitar el aprendizaje de contenidos y habilidades sociales, además de estar capacitado para poder entablar buenas relaciones comunicativas, forma parte esencial de las aptitudes y habilidades que dicho educador debe atesorar (LÓPEZ, 2005). 
Debe añadirse que incluso el niño o joven aún escolarizado al que se podría calificar de inadaptado necesita del educador social especializado con la finalidad de evitar la cronificación de su disconformidad y su desarraigo definitivo o, en su caso, de reconducir el disgusto enquistado y la deriva hacia la autoexclusión social.

Ese tipo de intervención educativa, que es complementaria de la educación formal propia del sistema educativo, es una acción pedagógica directa que actúa no solo sobre sujetos descontextualizados, sino sobre éstos en su propia realidad convivencial y existencial, es decir, sobre cada uno de ellos y sus familias en su propio contexto, en el barrio, en la escuela, sobre los grupos de pares y sobre la sociedad en su conjunto. En general, la intervención socioeducativa se estructura sobre tres principios fundamentales: el principio de prevención, el principio de desarrollo y el principio de intervención social (DE OÑA, 2009).

El primero de ellos, el principio de prevención, insta al educador social especializado a adoptar las medidas pertinentes para evitar, en la persona que necesita de su ayuda, el surgimiento o acrecentamiento de un acontecimiento, situación o estado negativo, ya sea reduciendo su probabilidad si aún no ha sobrevenido, evitando su agravamiento si es una realidad constatable, o buscando recomponer la normalidad perdida.

Por lo que se refiere al segundo principio que sirve de base a la intervención socioeducativa, el principio de desarrollo, hay que decir que consiste en la necesidad del acompañamiento educativo en cada una de las fases vitales por las que el sujeto pasa a lo largo de su vida; un acompañamiento cuyo objetivo es dotar a la persona que necesita de ayuda de aquellas instancias necesarias para que pueda observar, desentrañar y asumir lo más valioso de las experiencias nuevas y de los conocimientos venideros.

El principio de intervención social, o tercer principio de la intervención educativa que nos ocupa, supone una exigencia para el educador social especializado. Según ese tercer principio, el contexto social, que sirve de medio vital para el desarrollo personal de cada sujeto en situación vulnerable, de riesgo social o de exclusión, ha de poder ser comprendido y analizado por este en toda su complejidad. Solo así podrían posibilitarse procesos de extrañamiento cognoscitivo reparador y de reinserción saludable.

Se considera, pues, necesario y urgente apuntalar un proceso educativo que desarrolle de forma especial estrategias relacionales y diferenciadas capaces de contrarrestar la inercia 
socioeconómica marginadora de personas y de grupos, inercia que suele traducirse en abandono, delincuencia, depresión o drogadicción, entre otros efectos.

En efecto, el sistema educativo por sí solo no puede llegar a satisfacer las necesidades en dicho ámbito, y no solo por su uniformidad, sino además porque alberga un sector de la población aún no suficientemente lacerado por los procesos de exclusión social o sin problemas graves de adaptación social.

Corresponde a la educación social especializada el trabajo educativo de orientación con personas que se encuentran en situación social límite o de riesgo social. Son personas que, ya sea por ellas mismas o por el propio contexto en el que se insertan, sufren una existencia difícil de manera permanente e incluso de conflicto abierto consigo mismas, con sus familias o con el entramado social en su conjunto. La labor de tal intervención educativa consiste, pues, en potenciar el desarrollo de los recursos del sujeto excluido, o en situación de estarlo, con la finalidad de facilitarle su inserción social en sentido amplio y activo.

Dado que la tarea socioeducativa especializada se dirige a propiciar una búsqueda conjunta y específica, dialógica y concreta, de recursos actitudinales, éticos y sociales que mejoren la autoestima e incrementen el propio protagonismo en el proyecto vital de cada uno de los receptores de su actuación (MORATA, 2014), es necesario determinar cuáles son los medios pedagógicos fundamentales para llevar a cabo dicha tarea. Pues bien, dichos medios son el diálogo con el interlocutor, la cooperación con el destinatario y el fomento de la creatividad de este último por lo que se refiere a la búsqueda y consecución de soluciones.

La capacidad para realizar los análisis críticos que requiere el pedagogo social está ligada de modo específico a las aptitudes para la relación personal tanto como a la capacidad para trabajar en equipos interdisciplinares y multidisciplinares. Determinar las estrategias de intervención que atenúen o eviten el sufrimiento personal en los contextos de exclusión es importante (LOSADA; MUÑOZ; ESPIÑEIRA, 2015). Además, tan relevante como perentoria resulta la formación adecuada y las características personales que han de tener los educadores que se involucren profesionalmente en tales procesos.

Educação \& Formação, Fortaleza, v. 2, n. 6, p. 3-16, set./dez. 2017 


\section{LA INSTITUCIONALIZACIÓN DEL DEBATE POR EQUIPOS COMO METODOLOGÍA DOCENTE}

En general, el proceso de enseñanza y aprendizaje universitario no puede concebirse al margen de la promoción del desarrollo de estrategias que estimulen la formación cultural tanto como el desarrollo pleno de capacidades avanzadas del alumnado. En dicho contexto, resulta esencial la incorporación de nuevas metodologías docentes y de estrategias originales de mejora del acceso del estudiante universitario a los recursos bibliográficos y las fuentes de información generales, tanto materiales como virtuales.

Ahora bien, además de todo ello, se requieren procesos que generen el incremento de la capacidad de aprendizaje autónomo por parte del alumnado, la promoción de trabajo cooperativo, la mejora de las competencias lingüísticas y la puesta en práctica de las competencias de resolución de conflictos. A ello hay que añadir que entre estas capacidades cobran especial importancia tanto el desarrollo de la iniciativa personal como la aptitud para elaborar juicios razonados y análisis críticos.

La actividad docente aquí presentada consistió en institucionalizar sesiones de dialéctica y debate por equipos de estudiantes universitarios en el aula. Se fundó en una metodología activa y participativa. Así, la puesta en marcha de un proceso de debate por grupos o equipos entre estudiantes se propuso cubrir ocho objetivos fundamentales. Fueron los que a continuación se indican.

Lógicamente, el primero de ellos no podía ser otro que el de contar con la clara intención de institucionalizar, con carácter estable, en el aula sesiones de dialéctica y debate por grupos de estudiantes, mientras que el segundo fue el de promover el desarrollo de competencias lingüísticas y de habilidades comunicativas en los alumnos universitarios. El tercero fue instruir al alumnado en el desarrollo de la capacidad para fundamentar, exponer y defender tesis o posiciones, quedando en cuarto lugar la intención de suscitar el diálogo en el aula así como la capacidad de aceptación de los argumentos del adversario. El quinto de los objetivos fue el de motivar un clima de atención inteligente y activa, mientras que el sexto fue el de promover el trabajo en equipo. Y el séptimo y el octavo objetivo fueron potenciar la capacidad investigadora de los alumnos e intensificar el trabajo colaborativo concreto entre estudiantes y docentes. 
Se institucionalizó el debate en el aula desde el curso académico 2012-2013 hasta el 2015-2016, con la participación de una media de 230 alumnos por curso académico de distintas asignaturas del Grado de Pedagogía. La práctica de dicha metodología exigió que, tras la definición del tema a tratar, de las respectivas posiciones teóricas a sostener y de las tesis a argumentar, debía desplegarse un trabajo grupal continuo, colaborativo y bibliográfico, en el que la labor auxiliar del profesor hiciese su entrada en forma de asistencia y supervisión.

El despliegue del proyecto se realizó en cuatro fases. Durante la primera, se procedió al establecimiento conjunto de un código ético de comportamiento en los debates y de trabajo en equipo. La segunda fase consistió en la organización de los equipos y selección de las líneas temáticas. Por lo que respecta a la tercera fase, esta consistió en la determinación del tiempo asignado a cada intervención y selección de bibliografía. Y, en último lugar, se procedió a la selección de contenidos y al establecimiento del orden de intervención, de las fechas de las sesiones de debate y de la ejecución o implementación de estos.

El docente guió y supervisó la búsqueda de información de cada equipo, posibilitando abordar el debate con fiabilidad y rigor. Ello pasó por la recopilación, clasificación y selección de toda la información fidedigna referida al tema y ámbito de la pregunta propuesta. Dicha labor tutorial se llevó a cabo mediante recomendaciones sobre fuentes de información e incluso permitiendo la autonomía completa del alumnado en tal sentido tras señalar unas directrices iniciales de carácter formal.

Los parámetros de éxito de las sesiones de debate se fijaron conforme al grado de adecuación o inadecuación de las intervenciones con respecto a los siguientes aspectos: coherencia discursiva, diversidad y rigor de argumentos, precisión y variedad de las evidencias, agilidad en las respuestas, comunicación no verbal, naturalidad y, por último, utilización de recursos retóricos, tales como la adecuación al tono y momento del discurso. Todo ello ha ido orientado a enseñar, mejorar y consolidar las cualidades de los estudiantes de Pedagogía. Pues bien, por lo que se refiere a las cualidades estrictamente personales (SÁNCHEZ, 2011), debe señalarse que son cuatro las que se han considerado esenciales.

La primera de ellas es la posesión de criterios adultos; así, el educador social especializado debe ser un individuo independiente, flexible, perspicaz, sereno, seguro y crítico. En segundo lugar, y por lo que respecta a la madurez afectiva, el educador social especializado no 
puede ser un individuo desequilibrado, frustrado, violento, egoísta, susceptible, inseguro, narcisista, reprimido ni descontrolado. Por lo que se refiere a la capacidad de relación, dicho educador debe ser capaz de mostrar empatía, de poder actualizarse conforme a la experiencia y de expresarse adecuadamente en todas sus formas. En cuarto lugar, refiriéndose a la lucidez intelectual, el citado documento señala que el educador social especializado debe ser capaz de distinguir en los sujetos de su intervención lo consciente de lo inconsciente, las necesidades explícitas de aquellas que son implícitas. Por último, además, ha de saber interpretar adecuadamente las observaciones de la vida cotidiana e interpretarlas y ha de estar preparado para detectar el núcleo del conflicto que aqueja a la personalidad del sujeto atendido.

Junto a las citadas cualidades de su personalidad, el educador social especializado ha de contar con otras cualidades de carácter institucional (SÁNCHEZ, 2011). La primera de ellas es la capacidad de trabajo en equipo, la cual implica aptitud para la complementariedad, para generar y aceptar una ética intragrupal y, por último, para la participación activa. En segundo lugar, ha de ser capaz de mejorar la institución, destacando que ello implica tanto la capacidad de perseverancia como unos buenos fundamentos estratégicos y táctico-operativos, capacidad de resolución de conflictos y de cogestión de las actividades conducentes al desarrollo de las habilidades integradoras de carácter social e institucional. Por último, dicho educador ha de saber colaborar con el objetivo de intentar modificar las estructuras socioeconómicas que acentúan los déficits personales, y ello con la finalidad de conseguir que la sociedad facilite los procesos positivos de la correcta inclusión de las personas.

Para todo ello, resulta imprescindible la capacidad para fomentar actitudes sociales que permitan y fomenten el desarrollo comunitario de la persona excluida o en riesgo de exclusión, sustituyendo las actitudes de proteccionismo o de dominio por otras más justas e inclusivas.

\section{RESULTADOS PROCEDIMENTALES, COGNITIVOS Y ACTITUDINALES}

Los resultados obtenidos han confirmado una considerable y relevante evolución por lo que se refiere a los aspectos procedimentales, cognitivos y actitudinales que han de conformar la formación discente.

Educação \& Formação, Fortaleza, v. 2, n. 6, p. 3-16, set./dez. 2017

DOI: http://dx.doi.org/10.25053/edufor.v2i6.2379 
En cuanto al proceso metodológico seguido para recabar las evidencias, debe señalarse que en cada curso académico se elaboraron dos tipos de informe. Cada equipo de debate debía elaborar un informe de autoevaluación tras la finalización de las sesiones de debate. Además de ello, el profesorado debía elaborar su propio informe sobre la base de las actuaciones concretas de cada grupo y de cada uno de los miembros a lo largo de todo el proceso.

Dichos informes tuvieron un doble objetivo: por un lado, evaluar cada uno de los ítems que debían caracterizar la calidad de la preparación para el debate y de la ejecución de este; y, por otro lado, valorar las destrezas y la implementación de las capacidades formales, cognitivas y éticas mostradas por el alumnado en dicho proceso.

Por lo que se refiere a esas últimas, dichos informes se centraron en los siguientes apartados: solidez, oportunidad, relevancia y proyección de los argumentos y contraargumentos seleccionados; capacidad de argumentación; habilidades investigadoras; habilidades comunicativas; atención por la realidad; apertura analítico-cognoscitiva; consideración hacia la persona de los otros; respeto hacia las posiciones sustentadas por los otros; predisposición a aceptar tesis y argumentos convincentes distintos de los propios y, por último, despliegue de recursos conducentes al acuerdo y a la resolución dialogada de conflictos.

El análisis de los efectos formativos obtenidos y reseñados en los citados informes permitió constatar no solo un incremento en la concienciación autocrítica del alumnado, pero también una mejora del nivel autoevaluativo discente $y$, por último, el logro de una estimable mejoría por lo que se refiere tanto a los aspectos formales como al desarrollo del pensamiento analítico y a la mejora de la maduración personal.

Se prueba, pues, la eficacia de la puesta en práctica de las dinámicas aquí propuestas, pues estimulan la capacidad para trabajar en equipos interdisciplinares y mejoran e intensifican las aptitudes para la relación personal, la madurez, el equilibrio personal y la capacidad intelectual para el análisis de realidades personales y sociales.

\section{DISCUSIÓN}

Dada la insistencia de la comunidad científica en la tesis de que la formación universitaria de los educadores sociales debe exceder los límites de una mera formación aplicada 
(GARCÍA; SÁEZ, 2011), no se puede concebir su formación ni su desarrollo profesional al margen de la adquisición de capacidades reflexivas y éticas que ayuden a socializar al estudiante en el complejo mundo de la práctica profesional.

La realidad es que los futuros educadores deberán enfrentarse a la necesidad de pensar, decidir y actuar en el seno de equipos interdisciplinares y multidisciplinares. La presente iniciativa supone una novedad desde el punto de vista metodológico. Su carácter innovador estriba en probar los beneficios que la técnica metodológica del debate tiene en cuanto a la consecución de un mayor conocimiento, concienciación y formación procedimental y cognitivo-moral en el ámbito pedagógico preocupado por la exclusión social.

La hipótesis de partida consistió en tratar de evidenciar la utilidad de la práctica institucionalizada del debate por equipos en el aula para la cualificación del futuro pedagogo. Dicha hipótesis ha resultado probada tras el análisis de los datos obtenidos tanto de los procesos preparatorios como de la ejecución propiamente dicha y los resultados subsiguientes. La presente iniciativa es, además, pionera, pues liga la tendencia metodológica universitaria del debate por equipos en la educación superior al sentido específico de la pedagogía del cuidado (PANCHÓN, 2011).

La comparación entre la lista de los parámetros de éxito de las sesiones de debate con los de las capacidades formales, cognitivas y éticas exigidas en la formación del educador social arroja una interrelación directa. El presente proyecto se ajusta conceptual y procedimentalmente a los resultados vertidos por la literatura científica reciente sobre la formación del pedagogo vinculado a la acción social. La tesis inicialmente sostenida ha resultado, por lo tanto, corroborada en la práctica.

\section{CONTRIBUCIONES}

En sintonía con las exigencias recientes de la comunidad científica (PERICACHO, 2012), este proyecto presenta una doble contribución. En primer lugar, ha sistematizado e implementado una metodología que enfatiza la formación específica para abordar competencias esenciales. Tales competencias han sido el diálogo con el interlocutor, la cooperación con el

Educação \& Formação, Fortaleza, v. 2, n. 6, p. 3-16, set./dez. 2017

DOI: http://dx.doi.org/10.25053/edufor.v2i6.2379

http://seer.uece.br/redufor 
destinatario y el fomento de la creatividad de este último por lo que se refiere al logro de soluciones.

Con relación a la segunda contribución, ha mostrado la vinculación metodológica concreta entre la formación teórico-práctica y la práctica profesional. Ello se ha logrado poniendo el énfasis en estrategias formativas dirigidas a fomentar e instruir las siguientes características específicas: empatía, aptitudes para entablar relaciones personales sanas, capacidad para fomentar actitudes sociales, logro de un elevado equilibrio personal, capacidad de análisis de realidades personales y sociales, implicación en su labor y capacidad para trabajar en equipos.

La vía educativa elegida para intentar conseguir que las personas sensiblemente afectadas por situaciones personales, familiares o sociales perjudiciales puedan prevenir nuevas situaciones de riesgo, resarcir las dificultades presentes y reorientar la actividad futura, con el objetivo último de reconstruir y cimentar tanto su propia identidad como la conciencia de su valía personal, y puedan, simultáneamente, desarrollarlas en convivencia armónica con los demás ha sido la expuesta en la presente contribución.

Cabe concluir afirmando que la importancia social y humana que supone la intervención del educador social especializado ha de llevar necesariamente a un replanteamiento tanto de las concepciones sociales asumidas como de los fundamentos de las actitudes exteriorizadas. De ahí la relevancia de la formación en dicho ámbito, que ha de trascender la formación obligatoria y específica de los profesionales y del voluntariado social hasta llegar a impregnar la totalidad del tejido social.

\section{REFERENCIAS}

APOSPORI, E.; MILLAR, J. The dynamics of social exclusion in Europe: comparing Austria, Germany, Greece, Portugal and the UK. Cheltenham: Edward Elgar, 2003.

BELLVER, M. C. Prevenir la exclusión social. Valencia: Tirant lo Blanch, 2013.

CARMONA, G. Formación para la inclusión social. Nueva tendencia en la lucha contra la exclusión. Revista de Ciències de L'educació, Tarragona, n. 1, p. 87-103, 2005.

Educação \& Formação, Fortaleza, v. 2, n. 6, p. 3-16, set./dez. 2017

DOI: http://dx.doi.org/10.25053/edufor.v2i6.2379

http://seer.uece.br/redufor 
DE HAAN, A. Social exclusion: Towards an holistic understanding of deprivation. London: Department for International Development, 1999.

DE OÑA, J. M. Evaluación de un programa de educación social con jóvenes en situación de riesgo. Málaga: Spicum, 2009.

ESTIVILL, J. Panorama de la lucha contra la exclusión social: conceptos y estrategias. Ginebra: Oficina Internacional del Trabajo, 2003.

FERNÁNDEZ, F. Crisis, desigualdad, pobreza y exclusión. Berlín: Jornadas de Trabajo del Comité Europeo de Coordinación de Instituciones Nacionales de Derechos Humanos, 2013.

FISCHER, A. Reconceiving social exclusion (BWPI Working Paper 146). Manchester: University of Manchester-Brooks World Poverty Institute, 2011.

GARCÍA, J.; SÁEZ, J. Educación social: ¿Qué formación para qué profesional? Revista de Educación Social, España, n. 13, p. 1-14, 2011.

LÓPEZ, F. La educación social especializada con personas en situación de conflicto social. Revista de Educación, Madrid, n. 336, p. 57-71, 2005.

LOSADA, L.; MUÑOZ, J. M.; ESPIÑEIRA, E. M. Perfil, funciones y competencias del educador social a debate: análisis de la trayectoria de la formación de profesionales de la educación social. Educació Social: Revista d'Intervenció Socioeducativa, Barcelona, n. 60, p. 59-76, 2015.

MORATA, T. Pedagogía social comunitaria: un modelo de intervención socioeducativa integral. Educació Social: Revista d'Intervenció Socioeducativa, Barcelona, n. 57, p. 13-32, 2014.

PANCHÓN, C. Educadora social - Educador social: formación y profesión. Horizonte 2020. Revista de Educación Social, España, n. 13, p. 1-14, 2011.

PERICACHO, F. J. Exclusión y educación social: reflexiones en torno a su problematización y complejización. Revista de Educación Social, España, n. 14, p. 2-6, 2012.

SÁNCHEZ, C. Figura y formación del educador especializado, según el Centro de Formación de Educadores Especializados de Barcelona (CFEEB), 1976. Revista de Educación Social, España, n. 13, p. 1-13, 2011.

SILVER, H. The process of social exclusion: the dynamics of an evolving concept. (Chronic Poverty Research Centre Working Paper 95). Rhode Island: Brown University, 2007.

SUBIRATS, J. (Dir.). Análisis de los factores de exclusión social. Bilbao: Fundación BBVA, 2005.

TEZANOS, J. F. et al. En los bordes de la pobreza. Madrid. Biblioteca Nueva, 2013.

Educação \& Formação, Fortaleza, v. 2, n. 6, p. 3-16, set./dez. 2017

DOI: http://dx.doi.org/10.25053/edufor.v2i6.2379

http://seer.uece.br/redufor 
TSAKLOGLOU, P.; PAPADOPOULOS, F. Aggregate level and determining factors of social exclusion in twelve European countries. Journal of European Social Policy, London, v. 12, n. 3, p. 211-225, 2002.

Recebido em 20 de março de 2016.

Aceito em 7 de dezembro de 2016. 